Obtención de un patrón cromático digital para ser utilizado en la mejora genética por selección masal de la variedad Half BlackBlue o Diamante del pez ornamental Guppy (Poecilia reticulata; Peters, 1859)

\title{
Genetic improvement of variety pattern chromatic Half Black- Blue or Diamond of ornamental fish Guppy (Poecilia reticulata; Peters, 1859) through mass selection
}

\author{
Carlos Scotto Espinoza ${ }^{1}$ \\ ${ }^{1}$ Laboratorio de Mejora Genética y Reproducción Animal de la Facultad de Ciencias Naturales y Matemáticas. \\ Universidad Nacional Federico Villarreal. Jirón Río Chepén s/n. El Agustino. Lima. Perú.
}

Recibido 22 mayo 2011; aceptado 30 agosto 2011

\begin{abstract}
Resumen
El presente artículo determina un patrón digitalizado computarizado basado en la cantidad de píxeles. Se encontró ocho tonos de color azul característico tanto en el cuerpo como en la aleta dorsal para ser utilizado en el potencial mejoramiento genético por selección masal del patrón cromático de la variedad comercial Half Black-Blue o Diamante del pez ornamental Guppy (Poecilia reticulata). De diez parejas de padrillos escogidos con un promedio de ochenta crías F1 por cada pareja (Total $=734$ ), se seleccionaron al azar los doce animales machos más atractivos por su belleza cromática subjetiva, dividido en dos grupos, uno de color azul y otro de color celeste para encontrar un patrón digital cromático matemático por el número de píxeles.

Palabras clave: Poecilia reticulata, guppy, patrón cromático, píxeles.
\end{abstract}

\begin{abstract}
This article provides a standard computerized digitized based on the number of pixels. They found eight characteristic blue color tones in both the body and dorsal fin to be used in the potential for mass selection breeding color pattern of the commercial variety or Half Black-Blue Diamond ornamental fish Guppy (Poecilia reticulata). Ten pairs of sires selected with an average of eighty F1 offspring per couple (Total = 734), were randomly selected twelve male animals more attractive for its beauty subjective color, divided into two groups, one blue and one to find a light blue color digital mathematical pattern by the number of pixels.

Keywords: Poecilia reticulata, guppy, mass selection, pixels.
\end{abstract}

\section{Introducción}

Una variedad es un conjunto distinto, homogéneo y estable dentro de una especie animal. Una definición más extensa aclara que una "variedad" debe definirse por medio de sus caracteres (fenotipo) resultantes de un cierto genotipo (clon, línea, híbrido F1) o de una cierta combinación de genotipos (híbrido complejo, variedad sintética) claramente distintos de los de cualquier otra variedad por la expresión de al menos uno de dichos caracteres, y que además se mantendrán inalterados a través del proceso de reproducción (UPOV, 2008). Para la FAO (2011) el término "raza" comprende igualmente cepas, variedades y líneas de investigación. Una raza es un grupo homogéneo, subespecífico, de animales domésticos que poseen características externas definidas e identificables que permiten distinguirlos a simple vista, de

\footnotetext{
* Autor para correspondencia

Email: carlosscotto@yahoo.com (C. Scotto)
} 
otros grupos definidos de la misma manera en la misma especie; también es un grupo homogéneo sobre el que, debido a la separación geográfica con otros grupos fenotípicamente similares, existe un acuerdo general sobre su identidad separada (Turton, 1974).

\section{El pez ornamental Guppy (Poecilia} reticulata) es oriundo de la selva del Orinoco y de las islas de Trinidad y Tobago en América del Sur. Entre 1853 y 1859, zoólogos de Francia y Alemania empezaron a criar éste pez en acuarios europeos. En 1920, fueron exportados a los USA como pez tropical llegando a seleccionarse diferentes variedades en 1950 (Hidalgo, 1990). Constituyéndose con el tiempo en uno de los peces más populares a nivel Mundial. Actualmente, con diversas condiciones ambientales, métodos de alimentación y un control en el mejoramiento genético por selección se han obtenido guppys de mejor calidad principalmente en el patrón de color y en el tamaño corporal (Brooks y Endler, 2001). Existen más de 40 líneas de guppies clasificados por el color y para lo cual existe cinco tipos distintos de células pigmentarias o cromatóforos, clasificados en dos grupos, los que absorben luz, como son los melanóforos que proporcionan los colores negro y marrón, los eritróforos que dan el color rojo, y los xantóforos que dan el color amarillo y los que reflejan la luz como son los leucóforos que es blanco opaco y los iridóforos que dan un color plateado (Bagnara y Hadley, 1973).

Se ha determinado que el color gris (silvestre) está gobernado por un alelo dominante $(\mathrm{w})$ siendo dominante sobre otros alelos que determinan el color en distintas variedades de guppy como son el dorado, rubio, crema y el albino (Goodrich et al., 1944). Por otro lado, los colores corporales de las variedades Negra y Roja son dominantes sobre el color Amarillo y pueden presentar epistasis sobre otros colores más claros. El color negro puede abarcar todo el cuerpo o parte de él (1/4, 1/2 o 3/4), probablemente por factores influenciados por el sexo. Además, se ha comprobado que la "presencia de puntos negros en la cola" de las hembras es recesiva con respecto a la ausencia de los mismos (Rodríguez, 1980).

Se han descrito 18 genes del color en guppys, nueve están en el cromosoma Y, y ocho en el cromosoma $\mathrm{X}$, de los cuales tres no se recombinan con el cromosoma $\mathrm{Y}, \mathrm{y}$ cinco si lo hacen de $\mathrm{Y} \rightarrow \mathrm{X}$, de $\mathrm{X} \rightarrow \mathrm{Y}$ o de $X \rightarrow X$. Sólo un gen del color está en un cromosoma autosómico y controla el color del fondo del cuerpo. Además, cada gen presentaba un sistema multialélico particular de 24 alelos distintos que hace más difícil el análisis del mecanismo genético (Khoo, et al., 1999a; Khoo, et al., 1999b; Phang et al., 1989a; Phang et al., 1989b; Phang et al., 1990; Phang, V. \& Fernando, 1991; Phang, et al., 1999). El color Half Black (Factor HB) o también denominado "mitad negro", se caracteriza porque la parte posterior del cuerpo del pez es de color negro y ligeramente con reflejo azulado, y la parte caudal puede tener otro color como es el celeste, amarillo o rojo en tonos intensos o ligeros (pastel) con tendencia al verde o celeste. En la mayoría de los casos, el factor $\mathrm{HB}$ está en el cromosoma X. La mayoría de mejoradores de ésta línea cruzan un macho HB Blue con una hembra Blue para obtener tanto machos Blue como hembras Blue (F1). Los criadores descartan a todos los machos indeseados que escapan al patrón de $\mathrm{HB}$, y las hembras F1 son nuevamente cruzadas con el macho parental inicial para obtener animales superiores a la línea parental. Una segunda forma es cruzar machos y hembras HB Blue y descartar los que no salgan HB Blue, seleccionar dos grupos a parte de un macho de éste cruzamiento y aparearlo con otras hembras HB Blue, y de éste modo se podrán utilizar machos $\mathrm{y}$ hembras alternadamente de cada grupo para obtener crías sin llegar a tener mucha consanguinidad (Alderson, 2001). 
La Mejora Genética Animal es la aplicación de la ciencia genética a los rendimientos o la productividad, la resistencia a agentes abióticos y bióticos adversos, la belleza, la calidad o el rango de adaptación de los animales domésticos. El objetivo último es conseguir "tipos genéticos deseados" de animales que hagan lo más eficaz posible el sistema de producción del que son un componente esencial. La realización de un mejoramiento genético dirigido se basa en la gran variabilidad genética inicial de la población, asegura la existencia de la variabilidad suficiente para alcanzar las mejoras deseadas en las generaciones sucesivas. Después de la constatación de esta variabilidad se adopta una metodología de selección dirigida, con la identificación de todos los mejores reproductores y se elige a los que presentan la tasa de crecimiento más elevada para los cruzamientos sucesivos y la mejora del desempeño (Falconer, 1989).

La imagen digital ha revolucionado a la fotografía al hacerla más accesible que nunca. Hoy cualquiera puede participar del arte de la fotografía. Hoy existe la posibilidad de utilizar software de edición digitales de acceso libre que permiten perfeccionar o analizar en una computadora las imágenes obtenidas con una cámara digital a través de sus píxeles (unidad mínima de expresión gráfica).

Todas las cámaras ofrecen dos características para conseguir luz adecuada: la velocidad del obturador y la apertura del diafragma. La apertura del diafragma controla el tiempo de exposición a la luz. Cuanto más tiempo esté abierto el obturador más luz entrará. Por otro lado, cuanto más abierto éste el diafragma, más luz entra. Cuando queremos capturar imágenes en movimiento es importante utilizar un tiempo de disparo corto (imágenes congeladas) (Anónimo, 2006). Como muy bien señaló el artista Josef Albers (1998), el color es uno de los conceptos más relativos en el arte. No es la materia la que posee el color, sino que éste es una percepción sensorial.

La constitución molecular del objeto permite que absorba y refleje determinadas longitudes de onda. Este razonamiento es comprensible si tomamos en cuenta una serie de variables que inciden directamente en la percepción que tenemos de los colores y que pueden diferenciarse en tres categorías: (a) La fuente luminosa: gracias a la presencia de la luz se percibe no sólo los objetos sino también su cromaticidad. De las diferencias e intensidades lumínicas que inciden sobre el objeto resultan variaciones en la percepción de un mismo color. Existe, por ejemplo, diferencia entre luz solar y luz artificial; y dentro de la luz natural, las diferentes posiciones del Sol a lo largo del día hacen que la incidencia de luz provoque variaciones en el color del objeto. (b) El objeto: las características texturales de los objetos como transparencia, opacidad y brillo, entre otras, así como la forma y el tamaño inciden en la percepción del color. (c) El sujeto, en este punto se hace referencia al tipo de observador. No todos los animales perciben los colores como lo hace el ser humano (Anónimo, 2007).

En el siglo XVIII Isaac Newton estudió la luz a partir de sus observaciones del arco iris natural. Con la ayuda de algunos prismas y lentes, sobre los que hizo incidir la luz del Sol, Newton separó los colores con el fin de poder estudiarlos. Obtuvo así una banda de colores que denominó espectro solar. De ésta forma lo que podemos ver es el espectro de seis colores: rojo, naranja, amarillo, verde, azul y violeta. Tres son primarios: el rojo, el verde y el azul. El proceso de combinación de los colores primarios recibe el nombre de síntesis aditiva, de tal forma que, combinando los tres colores principales en proporciones e intensidades variables, se pueden obtener los demás colores. De ahí que el sistema de colores más usado en 
comunicación visual se llame RGB (iniciales de red, green y blue). Con ese proceso de síntesis aditiva todos los colores son posibles. Cada uno de nosotros ve los colores de una manera diferente; por lo cual, se han creado sistemas (representación gráfica) y modelos de colores para identificar el tono $\mathrm{o}$ combinación de un color. A lo largo de la historia, diversos investigadores han intentado ordenar el color de varias maneras, ya sea en forma bidimensional o tridimensional, tomando en cuenta las distintas variables. Una de las formas de organización en el plano más conocida es la utilización de un círculo llamado Círculo Cromático o mediante la Escala Acromática de grises que es una modulación continua del blanco al negro (Anónimo, 2004). La Comisión Internacional de la Iluminación eligió 3 colores primarios (Tricomia) con el fin de sentar una normativa universal que permitiera definir todos los colores espectrales. Dichos primarios corresponden a: Rojo $(700 \mathrm{~nm})$, Verde $(546 \mathrm{~nm})$ y el Azul (435 nm). El rojo es obtenido con lámpara incandescente $\mathrm{y}$ un filtro rojo normalizado, en cambio el verde y el azul son obtenidos con el arco de mercurio (Albers, 1998).

Así, el objetivo de este trabajo de investigación fue mejorar por selección genética el patrón cromático corporal del pez ornamental Guppy (Poecilia reticulata) comercial utilizando parámetros digitales computarizados y realizar una selección masal estratificada para la obtención de crías F1 que sirvan como reproductores futuros dentro de un programa de mejora genética de esta variedad.

\section{Materiales y métodos}

Se realizaron diez cruzamientos dirigidos de la variedad Half Black-Blue o Diamante del pez ornamental Guppy (Poecilia reticulata) adquiridos al azar en acuarios locales de la ciudad de Lima (Perú) y llevados al Laboratorio de Mejora Genética y Reproducción Animal de la Facultad de Ciencias Naturales y Matemáticas de la Universidad Nacional Federico Villarreal. Donde fueron mantenidos en condiciones óptimas para la reproducción $\quad\left(25^{\circ} \mathrm{C} \quad \mathrm{y}\right.$ aireadas constantemente). Por cada cruzamiento se utilizó un macho HB Blue adulto y una hembra $\mathrm{HB}$ Blue virgen y adulta. Los animales fueron alimentados ad libitum con alimento seco y tubifex. Se realizaron los cruzamientos en diez peceras individuales para obtener las crías F1 por selección masal (un promedio de ochenta crías por pareja). Se evaluó a los reproductores de cada pecera por separado por un periodo de dos meses hasta que se produjo la primera parición.

Todas las crías F1 ( $\mathrm{n}=734)$ fueron separadas en dos peceras nodrizas de 400 litros (uno para hembras y uno para machos) bajo las mismas condiciones óptimas de temperatura y oxigenación por burbujeo y alimentados ad libitum con nauplios de artemia y alimento seco importado rico en carotenoides estándar para alevines ad libitum hasta cumplir los 4 meses de edad (adultez). Se evaluó cuantitativamente el número total de crías obtenidas por pareja y se separó por sexo las crías de cada uno de los diez cruzamientos al evidenciarse el dimorfismo sexual (361 hembras y 373 machos).

A los cuatro meses al llegar a la adultez, doce de los mejores individuos seleccionados por su belleza corporal subjetiva. Y divididos en dos grupos -seis de color azul y seis de color celeste- de ambos sexos de la F1 fueron fotografiados digitalmente para determinar un patrón digital cromático potencial (mayor color azul o color celeste). Para lo cual, se utilizó el programa de análisis de imágenes computarizado Adobe PhotoShop Elements 4.0 (Versión gratuita: 
www.adobe.com/products/tryadobe) que permitió obtener valores matemáticos del tipo y cantidad del color azul por pixeles. Este análisis digital permitió seleccionar a los mejores animales que tuvieron una mayor cantidad de color azul o celeste en el cuerpo y en la aleta caudal. Los cuales fueron destinados como plantel de reproductores mejorados.

La Figura 1 muestra el Flujograma de la forma en que se obtuvo el patrón digital cromático para el mejoramiento de la variedad HB Blue en peces Guppy.

Se utilizó una cámara digital Marca Canon (Modelo PowerShot A590 IS) de 10 megapixeles (10X) sobre un trípode y una pecera plana diseñada para el fotografiado de $15 \mathrm{~cm}$ x $15 \mathrm{~cm}$ y de $1 \mathrm{~cm}$ de espesor sobre un fondo de color blanco, para lograr un mejor contraste estándar con los colores del animal. Y a una velocidad de obturación de 1/1250 segundos, una apertura de $\mathrm{f} / 5.5$, una longitud focal de 24 $\mathrm{cm}$ y una sensibilidad de ISO 400. Las tomas se realizaron al mediodía con luz solar entre los meses de Octubre y Noviembre con cielo despejado. Así mismo se colocó la cámara a una distancia de 10 centímetros del objeto para su fotografiado. Las fotografías digitales fueron analizadas por el programa de computación Photoshop para evaluar matemáticamente sus píxeles en la opción RGB con 16 tonos de color (Figura 2); de los cuales se utilizaron sólo los 8 tonos de azul mayoritarios e individuales tanto del cuerpo y de la aleta caudal de cada animal (Figura 3) y representados por píxeles en el círculo cromático o en la escala acromática (Figuras 4 y 5), en un monitor de computadora tipo Super VGA (Súper Adaptador de Gráficos de Video) con Resolución Predefinida Admitida (pixeles pulgada) de 800 x 600 .

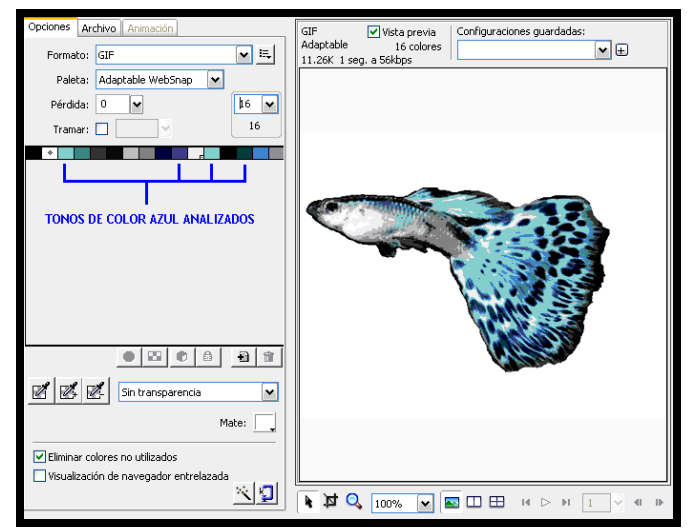

Figura 2. Análisis digital por el programa Photoshop de los píxeles de cuatro tonos de color azul mayoritarios presentes en el cuerpo del pez Guppy.

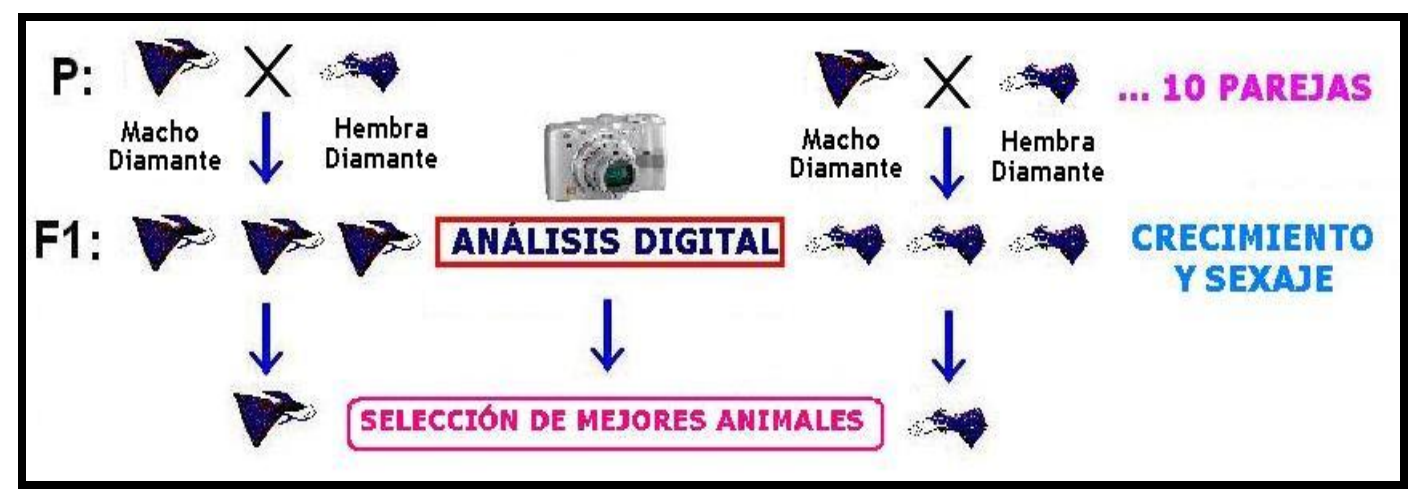

Figura 1. Flujograma para obtener crías F1 de diez apareamientos de parentales (P) de peces Guppys de la variedad Half-Black o Diamante y su análisis digital para obtener un patrón cromático a ser utilizado para seleccionar los mejores animales machos y hembras con el mejor patrón cromático como reproductores futuros. 


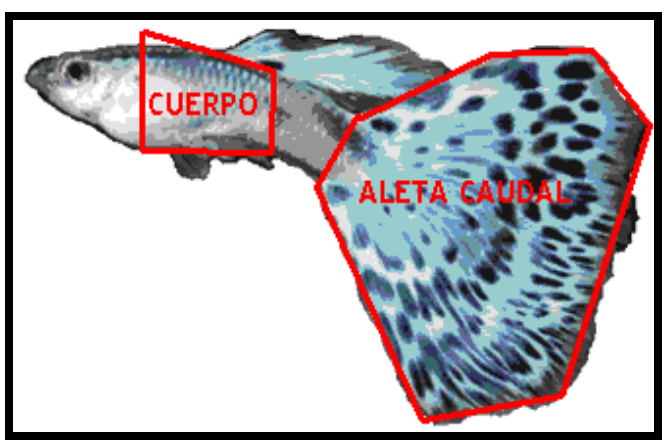

Figura 3. Áreas corporales analizadas digitalmente por el programa del pez Guppy (Cuerpo y la aleta caudal).

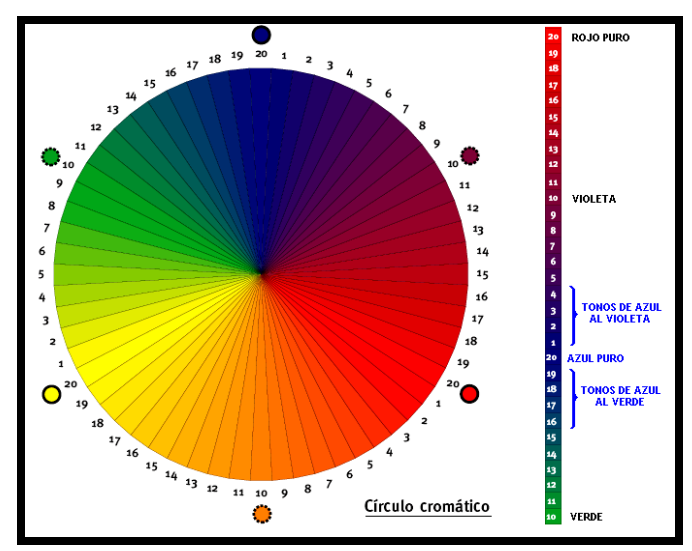

Figura 4. Círculo cromático mostrando el color azul analizado y sus tonos (Tomado de: http://www.wolkoweb.com.ar/apuntes/textos/circulo _cromatico.pdf).

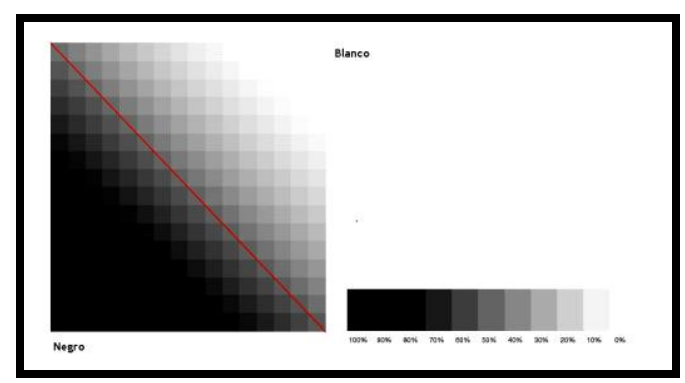

Figura 5. Escala acromática (Tomado de: http://www.wolkoweb.com.ar/apuntes/textos/circulo _cromatico.pdf).

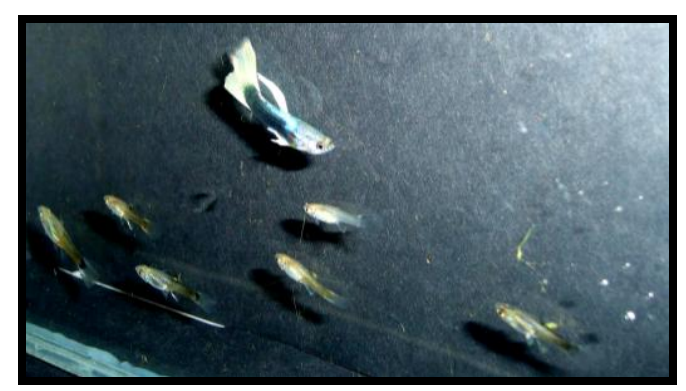

Figura 6. Cruzamiento de peces Guppys de la variedad Half-Black o Diamante (Parentales).

En la Figura 6 se observa el cruzamiento de machos Diamante con hembras Diamante vírgenes para obtener la F1 inicial para obtener el patrón cromático.

\section{Resultados y discusión}

En la Tabla 1 se muestra el patrón de los ocho colores mayoritarios encontrados en doce animales F1 selectos divididos en dos colores de cuerpo: azul y celeste. Ambos colores de cuerpo representan colores deseados comercialmente para venta en los acuarios peruanos debido a que alcanzan un mayor valor económico que los especímenes que no lo poseen. Es así que los animales machos de cuerpo azul presentaron en su cuerpo los cuatro tonos de coloración en el siguiente orden: 3, 2, 4 y 1 , cuyos valores en píxeles fueron respectivamente: 149, 116, 99 y 60 . Los cuatro tonos de coloración de la cola encontrados fueron: $3,4,2$ y 1 , cuyos valores en píxeles fueron respectivamente: 149, 99, 116 y 60. Los animales machos de cuerpo celeste presentaron tanto en su cuerpo como en su cola los cuatro tonos de coloración que fueron: 5, 8, 7 y 6, cuyos valores en píxeles fueron respectivamente: $165,153,148$ y 142. Los tonos mayormente presentes para los machos de cuerpo azul fueron los tonos 1 y 2 en el cuerpo, y los tonos 2 y 3 en la cola. Los tonos mayormente para los machos de cuerpo celeste fueron los tonos 7 y 8 en el cuerpo y los tonos 5,7 y 8 en la cola. 
Se encontraron seis tonos de colores presentes en la escala cromática que fueron los tonos 2, 3, 4, 5, 6 y 7. El tono 3 correspondió a un tono intermedio entre los colores azul y violeta. Mientras que los tonos 2, 4, 5, 6 y 7 correspondieron a tonos entre los colores verde y azul (Figura 4). Además, se encontraron dos tonos de color presentes en la escala acromática que fueron el Tono 1 (tendencia al color negro) y el Tono 8 (tendencia al color blanco) (Figura 5). La Figura 7 representa el tipo de animales con los colores propios de la variedad Diamante o HB Blue macho y hembra. Y las Figuras 8 y 9 representan animales machos y hembras de descarte cuyos patrón de colores no son los buscados.

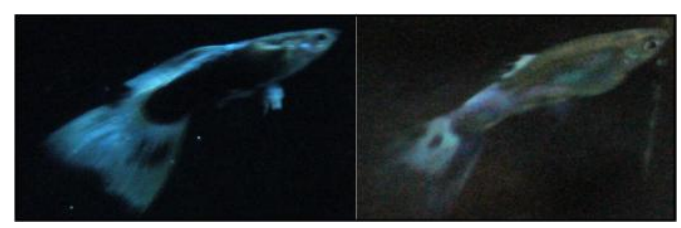

Figura 7. Macho parental con los colores propios de la variedad Diamante con cuerpo azul (aletas caudal y dorsal de color azul) (izquierda). Hembra parental con los colores propios de la variedad Diamante (aletas caudal y dorsal de color azul) (derecha).

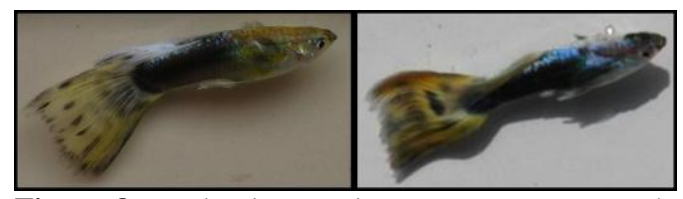

Figura 8. Macho descartado por presentar un patrón cromático inadecuado (caudal de color amarillo) (izquierda). Macho descartado por presentar un patrón cromático inadecuado (aleta caudal de color amarillo y tonos de color rojo) (derecha).

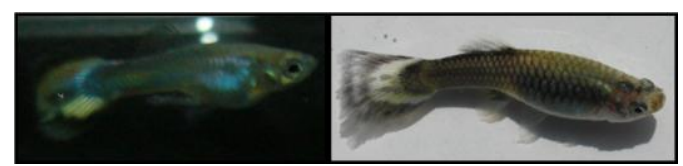

Figura 9. Hembras descartadas por presentar un patrón cromático inadecuado (aleta caudal con tonos de color amarillo) (izquierda y derecha).

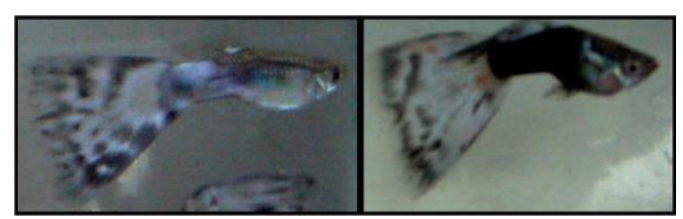

Figura 11. Machos F1 adultos con los colores propios de la variedad Diamante con cuerpo celeste (izquierda) y con cuerpo azul (derecha).

En la F1 se obtuvieron dos tipos de variantes de la Variedad HB, el de cuerpo Azul (Figura 11). Finalmente, se obtuvieron 12 animales selectos que fueron seleccionados como futuros reproductores (Figura 12).

La coloración del cuerpo de machos HB azules sería la mezcla de tonos de dos colores con tendencia al color verde (C2 y $\mathrm{C} 4$ ), un color con tendencia al color violeta (C3) y un tono gris con tendencia al color negro $(\mathrm{C} 1)$, mientras que la coloración de machos HB celestes, sería la mezcla de tonos de tres colores con tendencia al color verde (C5, C6 y C7) y un tono gris con tendencia al color blanco (C8).

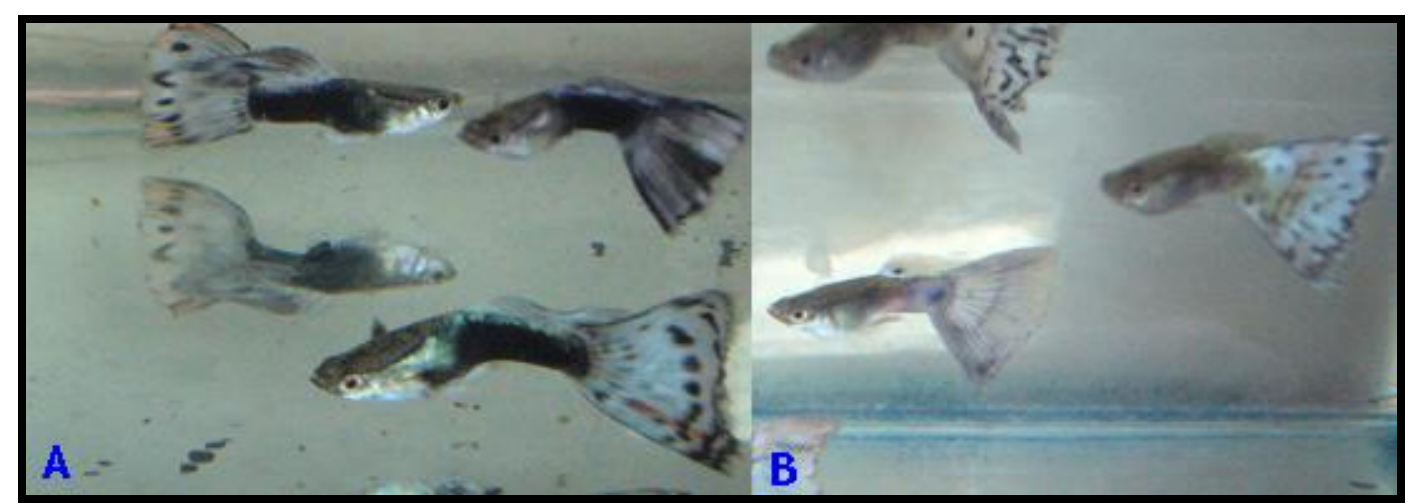

Figura 12. Machos F1 seleccionados como reproductores con los colores propios de la variedad Diamante con cuerpo de color azul (A) y con cuerpo de color celeste (B). 
Esto demostraría que la principal diferencia entre el color corporal entre ambos colores es la presencia del tono gris con tendencia al color negro (C1) para el HB azul, pero con el menor valor de 60 píxeles en comparación al resto de colores. La presencia del tono gris con tendencia al color blanco (C8) como segundo color predominante para el HB celeste, determinaría el viraje de los colores verdes predominantes hacia la coloración celeste encontrada en éste análisis.

En la Tabla 1 y Figura 13 se muestra el patrón de 8 colores mayoritarios encontrados en doce animales $\mathrm{F} 1$ selectos divididos en dos colores de cuerpo, azul y celeste.

\section{Tabla 1}

Número de píxeles obtenidos para cuatro tonos del color azul mayoritarios del cuerpo y de la cola de 12 animales machos selectos F1 del pez Guppy analizados por el programa Photoshop ( $\mathrm{A}=$ Número de Animal; $\mathrm{C}=$ Tipo de tono de color azul).

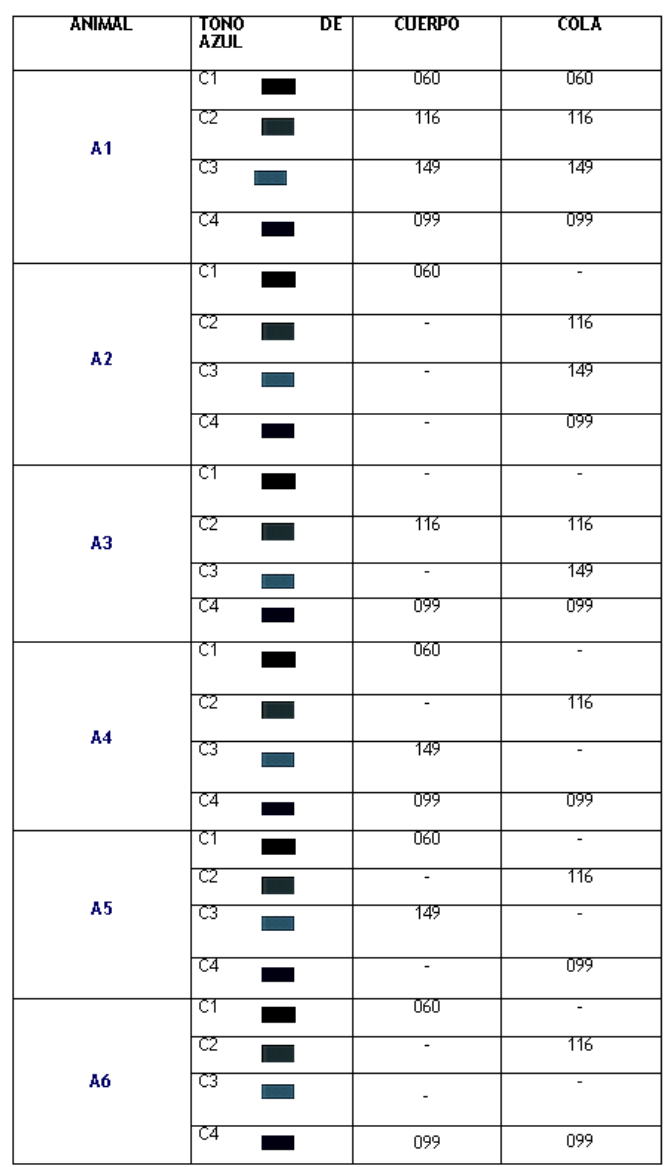

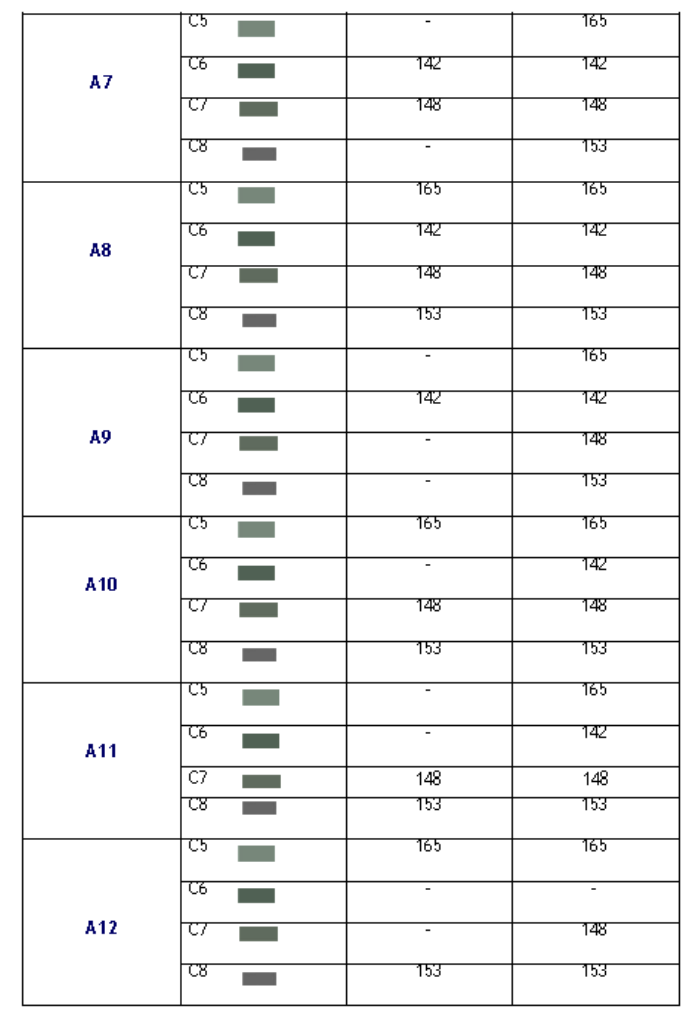

La coloración de la cola de machos HB azules sería la misma mezcla de tonos de dos colores con tendencia al color verde (C2 y C4), un color con tendencia al color violeta (C3) y un tono gris con tendencia al color negro (C1). Pero los tonos que mayormente se encontraron en la cola de la mayoría de machos fueron los tonos 2 y 4 , y no los tonos 1 y 2 como en el cuerpo. Siendo el tono 4 (C4) el que le da un color más tenue con tendencia al azul verdoso. Mientras que el tono $\mathrm{C} 1$ le da un color más oscuro y azulado al cuerpo.

Para la coloración de machos HB celestes, sería la misma mezcla de tonos que para el color del cuerpo: tres colores con tendencia al color verde (C5, C6 y C7) y un tono gris con tendencia al color blanco (C8). Sin embargo, se encontró en la cola mayormente el tono 5 adicionalmente a los tonos 7 y 8 encontrados también en el cuerpo lo cual atenuaría ligeramente aun más el color celeste de la cola con respecto al color del cuerpo del animal. 


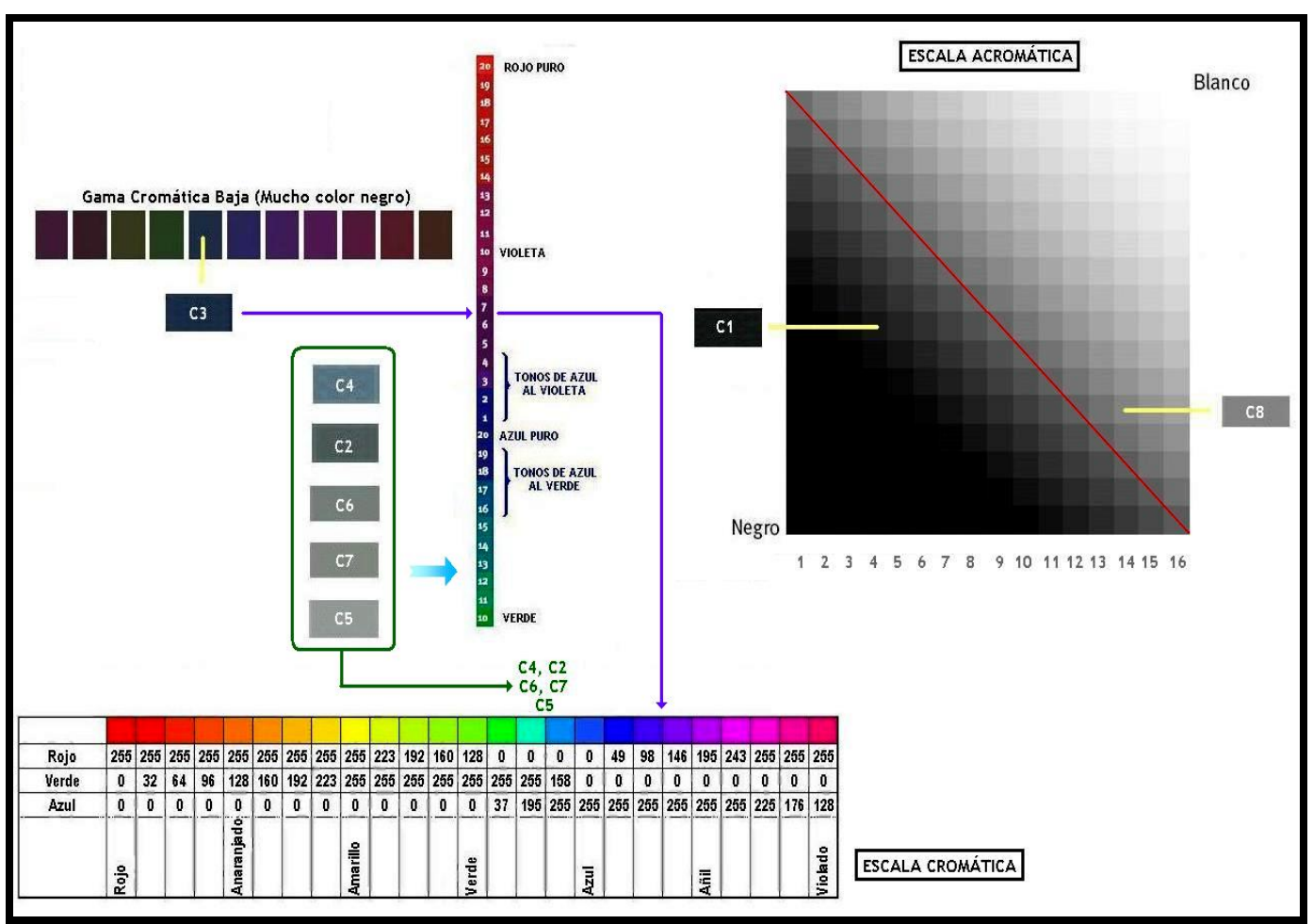

Figura 13. Clasificación de los ocho tonos de colores por píxeles mayoritarios (C1 al C8) encontrados en el cuerpo y la cola de guppys machos F1 selectos dentro de la escala acromática y cromática internacional (Tomado de: http://www.astropractica.org/tem2/ajucrom/LRGB.htm).

\section{Conclusiones}

Los animales machos de cuerpo azul presentaron en su cuerpo los cuatro tonos de coloración en el siguiente orden: Tono 3 $=\square>$ Tono $2=\square>$ Tono $4=\square>$ Tono $1=\square$, y cuyos valores en píxeles fueron respectivamente: 149, 116, 99 y 60. Los animales machos de cuerpo azul presentaron en su cola los cuatro tonos de coloración en el siguiente orden: Tono $3=$ $\square>$ Tono $4=\square>$ Tono $2=\square>$ Tono $1=\square$, y cuyos valores en píxeles fueron respectivamente: 149, 99, 116 y 60 . Los animales machos de cuerpo celeste presentaron tanto en su cuerpo como en su cola los cuatro tonos de coloración en el siguiente orden: Tono $5=\square>$ Tono 8 $=\square>$ Tono $7=\square>$ Tono $6=\square \mathrm{y}$ cuyos valores en píxeles fueron respectivamente: $165,153,148$ y 142. Los tonos mayormente presentes para los machos de cuerpo azul fueron los tonos $1 \mathrm{y}$ 2 en el cuerpo (presente en seis animales con cuerpo azul) y los tonos 2 y 4 en la cola (presente en seis animales con cuerpo azul). Los tonos para los machos de cuerpo celeste fueron los tonos 7 y 8 en el cuerpo (presente en seis animales con el cuerpo celeste), y los tonos 5,7 y 8 en la cola (presente en seis animales con el cuerpo celeste). Se encontraron seis tonos de colores presentes en la escala cromática que fueron $\operatorname{los}$ tonos 2, 3, 4, 5, 6 y 7 . El tono 3 correspondió a un tono intermedio entre los colores azul y violeta, mientras que los tonos 2, 4, 5, 6 y 7 correspondieron a tonos entre los colores verde y azul. Se encontraron dos tonos de color presentes en la escala acromática que fueron el Tono 1 (tendencia al color negro) y el Tono 8 (tendencia al color blanco). Se deben utilizar los tonos 1 y 3 para hacer selección del color en el cuerpo del HB azul. Para la coloración de la cola se deberá utilizar los tonos 3 y 4 . Se deben utilizar los tonos 5 y 8 para hacer selección del color en el cuerpo y la cola del HB celeste. 


\section{Referencias}

Albers, J. 1998. La interacción del color. Editorial Alianza Forma. Madrid.

Alderson, J. 2001. Linhagens Compativeis: o Blues e Half Black Blues. Boletin IFGA. Disponible en: http://www.kleberguppy.com.br/artigo6.htm.

Anónimo. 2006. Gran guía de fotografía digital. Edición Especial. National Geographic. ISSN 1665-7764.

Anónimo. 2007. Captura y mejora tus fotos digitales. PC Actual, Computer Idea.

Anónimo. 2004. El color, ese gran desconocido. Diseño Digital 1(12): 4-7.

Adobe PhotoShop Elements 4.0 (Versión gratuita). Disponible en: http://www.adobe.com/products/ tryadobe.

Bagnara, J.; Hadley, M. 1973. The nature of pigmentation. Ed. Englewood Cliffs, N.J.: Prentice-Hall Press. USA. p. 44-45.

Brooks, R.; Endler, J. 2001. Female guppies agree to differ: phenotypic and genetic variation in mate choice behaviour and the consequences for sexual selection. Evolution 55: 1644-1655.

Falconer, D. 1989. Introduction to quantitative genetics. 3rd ed. Ed. Longman, Essex, UK.

FAO. 2011. Disponible en: http://www.fao.org/docrep/ V8300S/v8300s0c.htm

Goodrich, H.; Josephson, N.; Trinkaus, J.; Slate, J. 1944. The cellular expression of two genes in Lebistes reticulatus. Genetics 29: 584-592.

Hidalgo, M. 1990. Cómo obtener guppys de calidad. Chalwa: Revista de Acuarismo 1(3): 15-21.
Khoo, G.; Lim, T.; Chan, W.; Phang, V. 1999a. Genetic basis of the variegated tail pattern in the guppy, Poecilia reticulata. Zoological Science 16: 431-437.

Khoo, G.; Lim, T.; Chan, W.; Phang, V. 1999b. Sexlinkage of the black caudal-peduncle and red tail genes in the Tuxedo strain of the guppy, Poecilia reticulata. Zoological Science 16: 629-638.

Phang, V.; Ng, L.; Fernando, A. 1989a. Inheritance of the snakeskin color pattern in the guppy, Poecilia reticulata. J. Hered. 80: 393-399.

Phang, V.; Ng, L.; Fernando, A. 1989b. Genetics of the color of the yellow snakeskin variety of the guppy, Poecilia reticulata. Singapore J. Pri. Ind. 17: 19-28.

Phang, V.; Fernando, A.; Chia, E. 1990. Inheritance of the color patterns of the blue snakeskin and red snakeskin varieties of the guppy, Poecilia reticulata. Zoological Science 7: 419-425.

Phang, V.; Fernando, A. 1991. Linkage analysis of the Xlinked green tail and blue tail color genes in the guppy, Poecilia reticulata. Zoological Science 8: 975-981.

Phang, V.; Khoo, G.; Sock-Peng, A. 1999. Interaction between the Autosomal Recessive bar Gene and the YLinked Snakeskin Body (Ssb) Pattern Gene in the Guppy, Poecilia reticulata. Zoological Science 16: 905-908.

Rodríguez, R. 1980. Lebistes. Revista Acuarama 3: 5-7.

Turton, J. 1974. The collection, storage and dissemination of information on breeds of livestock. Proceedings of 1st World Congress On Genetics Applied To Livestock Production, Madrid: 61-74.

UPOV. Unión Internacional para la protección de las obtenciones vegetales. 2008. Disponible en: http://www.upov.int/es/about/pdf/c_extr_19_2_rev.pdf 